\title{
Time for laughter
}

\author{
Francesca Bonin $^{\mathrm{a}, \mathrm{b}, *}$, Nick Campbell $^{\mathrm{a}}$, Carl Vogel ${ }^{\mathrm{b}}$ \\ ${ }^{a}$ Speech Communication Lab, Centre for Language and Communication Studies, School of Linguistic, Speech and Communication Sciences, Trinity College Dublin, University of \\ Dublin, Dublin 2, Ireland \\ ${ }^{\mathrm{b}}$ Centre for Computing and Language Studies, Computational Linguistics Group, School of Computer Science and Statistics, Trinity College Dublin, University of Dublin, Dublin \\ 2, Ireland
}

\section{A R T I C L E I N F O}

\section{Article history:}

Received 30 November 2013

Received in revised form 19 April 2014

Accepted 20 April 2014

Available online $\mathrm{xxxx}$

\section{Keywords:}

Social signals

Laughter

Topic change

Discourse analysis

Conversational analysis

TableTalk

AMI

\begin{abstract}
A B S T R A C T
Social signals are integral to conversational interaction and constitute a large part of the social dynamics of multiparty communication. Moreover, social signals may also have a function in discourse structure. We focus on laughter, exploring the extent to which laughter can be shown to signal the structural unfolding of conversation and whether laughter may be used in the signaling of topic changes. Recent research supports this hypothesis. We investigate the relation between laughter and topic changes from two different points of view (temporal distribution and content distribution) as visible in the TableTalk corpus and also in the AMI corpus. Consistent results emerge from studies of these two corpora. Laughter is less likely very soon after a topic change than it is before a topic change. In both studies, we find solo laughter significantly more frequent in times of topic transition than in times of topic continuity. This contradicts previous research about the social dynamics of shared versus solo laughter considering solo laughs as signals of topic continuation. We conclude that laughter has quantifiable discourse functionality concomitant with social signaling capacity.
\end{abstract}

(C) 2014 Elsevier B.V. All rights reserved.

\section{Introduction}

We begin with the observation that laughter is only sometimes purely the vocalization of mirth. One difference between unbridled mirth and controlled laughter may be in the internal structure of the laughter: controlled laughter does not exhibit random structure but repetitions; uncontrolled spontaneous laughter has been found to have random internal structure [1]. Some have sought to classify laughter according to the visual appearance and have found evidence in artworks sufficient to separate four types of laughter: joyful, intense, schadenfreude laughter, grinning [2]. It may be a response to what has preceded in conversation or in the external context of the conversation in which it appears. Laughter may also signal what is to follow in conversation, perhaps an explanation of the outburst. In a different dimension, laughter can be understood as a joint activity: one interlocutor may laugh alone, or a number may join the laughter. Previous authors [3] have described laughter as an action in its own right, the occurrence of which may be independent from the presence of humor. In this context, laughter has been seen as a highly ordered phenomenon, internally and externally. In this sense, it is also relevant to explore the timing of laughter with respect to

\footnotetext{
* Corresponding author at: Centre for Computing and Language Studies, Computational Linguistics Group, School of Computer Science and Statistics, Trinity College Dublin, Ireland. Tel.: +353018961718.

E-mail addresses: boninf@tcd.ie (F. Bonin),nick@tcd.ie (N. Campbell),vogel@tcd. ie (C. Vogel).
}

other elements of interaction in dialog. We wish to explore hypotheses about the differential signals effected by shared laughter and solo laughter in conversation. We think that the timing of mirthful laughter is effectively random, given the distribution of potential triggers. ${ }^{1}$ However, we believe that when laughter functions as a social signal, its timing is structured and conveys information about the underlying discourse structure. Previous works have explored other non-verbal features that can be predictive of discourse structure [5-7]. Luz et al. $[5,6]$ investigate the potential of non-verbal signals such as silences (among two speakers vocalizations as well as within the same speaker turn) and overlaps in predicting topic changes in meetings. Results show that pauses and overlaps on their own are good estimators of the topic structure of meetings conversation, reaching performance comparable with lexical based methods.

In this work, we extend a previous analysis of the TableTalk corpus $[8,9]$ to the AMI corpus [10]. ${ }^{2}$ Both corpora involve communication in English, where English is a lingua franca in one

\footnotetext{
${ }^{1}$ While we see distinction between instances of mirthful laughter and structural laughter we do not here seek functional (or automatic) discrimination nor attempt to understand speakers' emotive state (others, of course, do attempt to infer speaker emotions [4]); rather, we treat all instances of laughter as instances of the category 'social signals'.

2 Our research is anchored in available multimodal corpora. While the number of corpora available with annotations appropriate to our purposes is not vast, it is possible to note qualitative differences in two such possibilities and hold the results which obtain for them as representative of their types until more instances of those types can be annotated and studied, along with instances of other types, as well.
} 
setting and a native language in the other. Politeness dimensions to laughter in conversation might have different manifestations in the two corpora given other aspects. In the TableTalk conversations, recorded in Japan, the dialog includes five participants, sitting around the table, chatting. They included one native speaker of Japanese, one of Finnish, one of French (Belgian), and two native speakers of English (one Australian, one British). The Japanese participant and her Australian friend were rewarded for taking part in the conversation, while the others were visiting researchers in the lab directed by the native English speaker. This dialog had no particular structure, but tended to be around the theme of life in Japan (see Section 3.1). In the AMI corpus, participants are presumed to be unfamiliar with each other (at least they were recruited in that way), and paid to talk to each other for the data collection. The conversations in this corpus was structured as collaborative tasks (see Section 3.2). We take these corpora as exemplars because neither was constructed with the specific purpose of studying laughter.

In a previous study we analysed TableTalk [9] and showed a relation between laughter and topic changes in spontaneous conversations; laughter did not appear to be a random or exclusively content-driven event, but we detected a tendency for higher probability of laughter, particularly shared laughter, towards topic ends. Conversely, we found longer periods without laughter immediately after a topic change. Such findings support the hypothesis of the existence of a discourse function of laughter. In the same work, we analyzed laughter also with respect to the information flow. We distinguished two types of discourse segments and examine laughter as a discourse marker, signaling the onset of a topic termination segment [11], or the end of a topic-onset segment. We found that topic termination segments thus marked tend to have higher lexical variety than topic onsets.

Our present investigations are twofold. We extend our previous analysis and we explore on both corpora: (i) the temporal distribution of topic changes, (ii) the temporal distribution of laughter in structured and unstructured conversations, seeking to answer the following questions:

(1) Is there a pattern in the temporal distribution of laughter (and of shared and solo laughter)?

(2) How does information flow vary in topic termination and topic beginning segments?

The paper is structured as follows: an introduction is given in Section 1 . Section 2 provides operational definitions that will be used in the rest of the paper. Section 3 describes the two corpora, and Section 4 shows the correlation between frequency of laughter and topic changes. Experiments are described in Section 5. Section 5.1 answers question 1, and Section 5.2 answers question 2. Results are discussed in Section 6, and conclusions are drawn in Section 7.

\section{Definitions and measurements}

Understanding whether laughter has a function in the discourse structure plays a crucial role in the framework of discourse segmentation, as laughter could constitute an informative feature to boost topic segmentation efficacy. For the present work, we have considered topic at a discourse level characterized by a chunk of coherent content.

\subsection{Definition of topic}

A formal definition of "topic" is surprisingly difficult to provide (cf. "subject", [12]), as it is understanding where borders stand between topics and subtopics. Topic can be seen to cover different levels of granularity and different contexts. The linguistics literature has distinguished two levels of granularity: a sentence level [13], and a discourse level [14]. On the other hand, in the context of topic segmentation algorithms, topic has been mostly referred to at a discourse level, as segments of the discourse sharing coherent information (about the same thing [15]). Passonneau et al. [16], interpret topic as speakers' intentions, and topic changes in conversations as changes in the participants' activities (information-giving, decision-making). In topic segmentation applications, such as information retrieval from broadcast news, topics have been referred to as lexically coherent segments of the discourse [17], often having completely different themes. Many different topic segmentation algorithms have been developed on the basis of the lexical coherence approach described in [17]; others have exploited clustering approaches [18], others discourse markers that provides clues about the discourse structure [19], but few have tackled the difficult problems of casual conversational speech. In this work we consider topic a fragment of discourse about the same subject, relying on the topic annotation of the corpora at hand. Details on the topic annotation used in the present work are given in Section 3.3.

\subsection{Temporal definitions and measurement}

Laughter and topic boundaries serve as conversational landmarks. We work with an abstraction of topic changes (T-events) as instantaneous points of topic shift in conversation. We consider the laugh events in relation to T-events. First we explore the distance between laughter in general and T-events, looking at the time spans between the last laugh in topic A and T-event (namely LT) and the T-event and the first laugh in topic B (namely TL) (Fig. 1). Then, we analyze the behavior of types of laughter, shared vs. solo, with respect to T-events. In this case, our foci are the last solo (SO) and shared (SH) laughs prior to a T-event (named LL: SoLL or ShLL, respectively). See Fig. 2.

We denote the measure of the distance (in seconds) between Tevents and boundary laughs with $\mu$. Below we consider the differences between $\mu(\mathrm{LT})$ and $\mu(\mathrm{TL})$ as well as between $\mu(\mathrm{SoLT})$ and $\mu$ (ShLT).

Finally, we concentrate on the distinction between topic continuation moments and topic transition moments, analyzing the distribution of laughter among those segments. We construct operational models of topic continuation segments, calling them wi segments, and topic transition segments, calling them wo segments. We define these as follows (see Fig. 3):

- wi segments: the central half of each topic;

- wo segments: the final quarter of one topic and first quarter of the next topic;

By construction, wi segments represent the core of a topic and have topic cores within them, while wo segments do not contains the core of a topic, but do contain a transition between two topics. Both are defined in relation to the duration of a sequential pair of topics, not absolute durations. We find this decomposition of

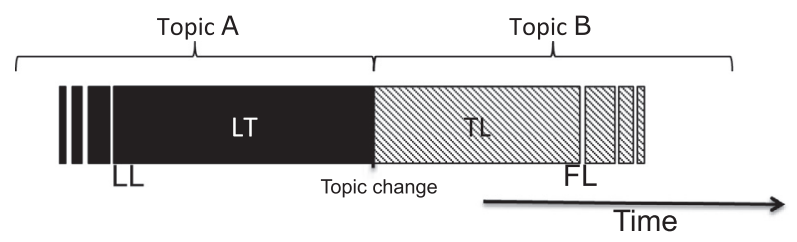

Fig. 1. Topic boundary neighborhood. LL and FL represent last and first laugh. LT and $\mathrm{TL}$ represent respectively a topic termination segment and a topic beginning segment. 

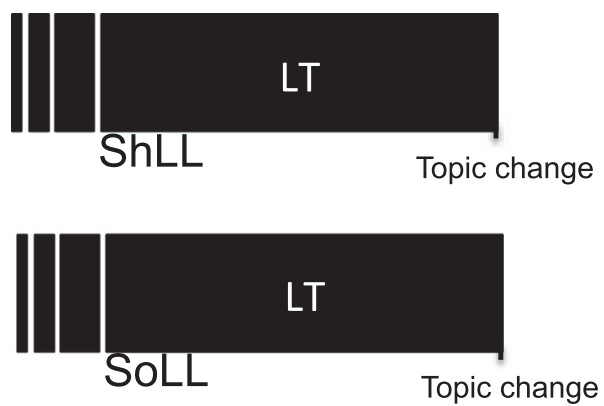

Fig. 2. Topic boundary left neighborhood with shared and solo last laughs (ShLL and SoLL).

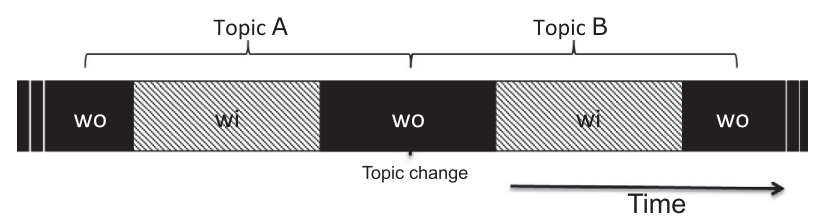

Fig. 3. Topic continuum vs. topic transition segmentation.

conversational flow into segments of topic-core talk and topic transitions to have face validity, in the sense the term is used in psychology to indicate that the objects used operationally relate naturally to the corresponding theoretical constructs.

\subsection{Content analysis and measurement}

In order to answer (2), we measure LT and TL segments of the conversation for their lexical richness. We take variation in lexical richness as a proxy measure of information flow at the onset of the topic and at the end of the topic (onset and end determined by a social signal, i.e. laughter, rather than some lexical indicator). We refer to the lexical richness of LT and TL as $\Lambda$, respectively $\Lambda$ (LT) and $\Lambda(\mathrm{TL})$.

For each T-event, $t$, we define $\Lambda\left(\mathrm{LT}_{t}\right)$ as:

$\Lambda\left(\mathrm{LT}_{t}\right)=\operatorname{TTR}\left(\mathrm{LT}_{t}\right) /$ Length $\left(\mathrm{LT}_{t}\right)$

where TTR is the Type/Token ratio. Similarly for $\Lambda\left(\mathrm{TL}_{t}\right)$.

\subsection{Shared laughter annotation}

In order to analyze the dynamics of shared and solo laughter in both corpora, an annotation of whether a laugh is an isolated one or a shared one is necessary. TableTalk and AMI do not provide such detailed annotation. Hence, we developed a novel strategy for shared laughter annotations.

In previous work [9] we defined shared laughter as overlapping laughs or consecutive laughs within $1 \mathrm{~s}$ distance. It was based on the intuition that consecutive laughs, if separated by a small enough distance would still be experienced and externally perceived as shared. This threshold was experimentally determined without the existence of a gold standard to refer to. Here we test an extreme position that only truly overlapping laughter is to be regarded as shared. Therefore, in the current work, we consider shared co-occurrent laughter of different speakers, where cooccurrent indicate overlapping as well as successive laughter with no gap between them. The reason for this stand in investigating a baseline situation in which, in order to be defined as a shared laugh, a laughter has to overlap or occur sequentially without an intervening gap.
We extend this annotation to the entire TableTalk and AMI. However, as has been noted by others [20], the annotations of the temporal aspects of laughs in AMI are partly flawed. Many instances (c. 25\%) of laughter have start points that coincide with terminations, resulting in a zero duration. We decided to focus our analysis on the laughs having start time different from end time. $^{3}$

\section{Corpora}

As mentioned above, analyses are based on two datasets of different nature. The characteristics of the two corpora allow us to compare human interactions in several situations: free natural interaction (TableTalk) and more structured task-based interactions (AMI).

\subsection{TableTalk}

TableTalk $^{4}$ is a corpus of free flowing natural conversations, recorded at the Advanced Telecommunication Research Labs in Japan (see Fig. 4). It is a multi-modal corpus of conversations among five individuals [8]. In order to collect as natural data as possible, neither topics of discussion nor activities were restricted in advance. The recordings were made in an informal setting over coffee, by three female (Australian, Finnish, and Japanese) and two male (Belgian and British) participants. A more complete description of the recording setup can be found in [21]. The recordings are carried out over three sessions, of different lengths ranging from $35^{\prime}$ to $1 \mathrm{~h}$ and $30^{\prime}$, recorded on consecutive days. The conversations are fully transcribed and segmented for topic, and also annotated for affective state of participants and for gesture and postural communicative functions using MUMIN [22]. Table-talk has been analyzed in terms of engagement and laughter [9,23-25] and lexical accommodation [26]. Our analyses used transcripts of the entire corpus: about $3 \mathrm{~h}$ 30, 31,523 tokens and 5980 turns. Laughter was transcribed in intervals on the speech transcription tier as @w, (unless inserted as part of a longer utterance). The total number of laughs is 713 . Other annotations are topic information (see 3.3) and emotional state of the participants.

The five participants present different features: three of them are researchers and two of them are rewarded participants. On a different dimension, participants also differ with respect to native language, English language skills, and culture. Subgroups are present also with respect to the acquaintance between them: two among the researchers (the English and the Belgian) knew each other before setting up the experiment. The same for the Japanese and the Australian. A detailed study of the relation between participants and its reflection over individual vs. group engagement is reported in [23].

Table 1 reports the amount of different laughter per speaker. For the lexical analysis, the transcripts have been processed using the Stanford PoS Tagger [27].

\subsection{AMI}

The AMI (Augmented Multi-party Interaction) Meeting Corpus is a multi-modal data set consisting of $100 \mathrm{~h}$ of meeting recordings [10]. The dataset is derived from real meetings, as well as scenariodriven meetings, designed to elicit several realistic human behaviors (see Fig. 5). We base our analysis on the scenario based meetings, for a total of 717,239 tokens, relying of the

\footnotetext{
${ }^{3}$ Given the existence of annotation flaws, in the future, we intend to investigate possible robust solutions for shared laughter annotation, that exploit only the starttime information of the laugh instances.

${ }^{4}$ Freely available at: http://sspnet.eu/2010/02/freetalk.
} 


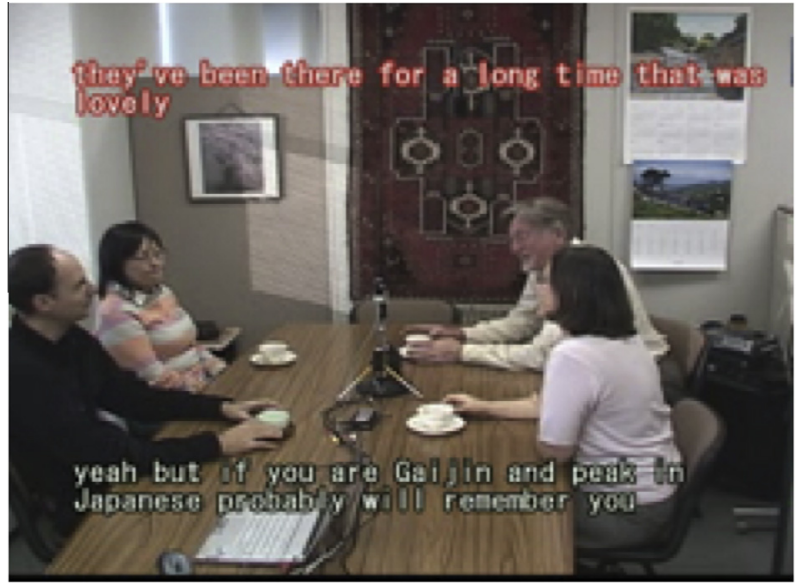

Fig. 4. TableTalk screenshot.

Table 1

Distribution of laughter among speakers - *Speaker g participated only in Day 2.

\begin{tabular}{lccc}
\hline Speaker & Shared & Solo & Total \\
\hline $\mathrm{d}$ & 33 & 5 & 38 \\
$\mathrm{~g}_{*}$ & 20 & 9 & 29 \\
$\mathrm{k}$ & 138 & 56 & 194 \\
$\mathrm{n}$ & 175 & 59 & 234 \\
$\mathrm{y}$ & 162 & 56 & 218 \\
\hline
\end{tabular}

conversations' transcriptions. Each meeting has four participants, and the same subjects meet over four different sessions to discuss a design project. The sessions correspond to four different project steps (Project kick-off meeting, functional design, conceptual design and detailed design).

Each participant is given a role to play (project manager, marketing expert, industrial designer and user interface designer) and keeps this role until the end of the scenario. Conversations are all in English, but participants are not all English native speaker (91 over 187 are English native speakers, the rest is divided between other 27 nationalities. ${ }^{5}$ ) Table 2 indicates the average number of laughs per speaker. There are 11,277 instances of laughter, and they are annotated in the transcripts as "vocal-sounds/laugh".

\subsection{Topic annotation in TableTalk and AMI}

For both corpora (TableTalk and AMI) we rely on the manual topic annotation provided. In TableTalk, topics have been annotated manually by two labelers at a coarse level and no distinction is made between core topics and subtopics. AMI provides the annotation of top-topics and subtopics. Top-level topics refer to topics whose content reflects the main meeting structure, while subtopics reflects small digressions inside the core topics. For this analysis we have focus on the core topic segmentation which seemed to be more in line with the TableTalk annotation.

\section{Laughter \& topic probability distribution}

We imagine a conversation as a flow where the probability of laughter as well as the probability of changing topic may vary over time. There will be moments in the conversation where the interaction between participants is more dynamic and the dis-

\footnotetext{
${ }^{5}$ Arabic, Chines, Chinese, Czech, Czeque, Dutch, English, Estonian, Finnish, French, German, Greek, Hindi, Italian, Konkani, Malayalam, Mandarin, Persian, Polish, Portuguese, Russian, Spanish, Swedish, Swiss, Tamil, Telugu, Vietnamese, Wolof, Romanian.
}

Table 2

Average distribution of laughs per speaker in the AMI corpus.

\begin{tabular}{llr}
\hline & Avg. & \multicolumn{1}{c}{ SD } \\
\hline Shared & 25.46 & 19.03 \\
Solo & 48.89 & 41.29 \\
Tot. (Sh + So) & 81.8 & 105.13 \\
\hline
\end{tabular}

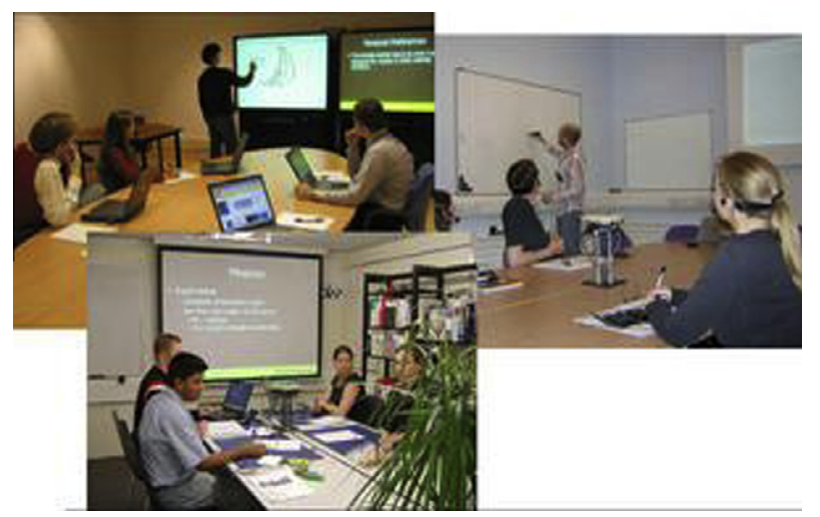

Fig. 5. AMI screenshot - ㄷ AMI website: http://https://www.idiap.ch/dataset/ami/.

course more unstructured, as well as moments in the conversation in which the discourse will tend to be more structured. We imagine the former being moments of high entropy characterized by shorter topics (hence more topic changes), and the latter being moments of lower entropy, characterized by longer topics and fewer topic changes. We are interested in exploring the correlation between those moments in the conversations and the presence of laughter. In particular, whether there is a relation between moments of the conversation with higher number of topic changes and more laughter. To this aim we segment the conversations in progressive windows of $240 \mathrm{~s}^{6}$ and we calculate the amount of laughter and of topic changes per window.

Looking at the two distributions (laughs per windows and topic changes per window), we notice a positive correlation between the mean frequency of laughter per window and the mean frequency of topic change per window. Fig. 6 shows the linear correlation (Pearson correlation test $=0.6$ ) between these distributions: windows with more topic changes correspond to windows with higher number of laughs.

The evidence of this correlation suggests that topic changes and laugher are linearly correlated. Therefore, we investigate this correlation in terms of timing.

\section{Experiments}

\subsection{Laughter \&' topic: temporal distributions}

In our first analysis we attempt to understand whether there is a pattern in the temporal distribution of laughter with respect to topic changes in the analyzed corpora. To this aim, we conduct two experiments:

$\mathrm{E}_{1}$ : we examine the left (LTs) and right sides (TLs) of topic boundaries considering $\mu(\mathrm{LT})$ and $\mu(\mathrm{TL})$ (Fig. 1).

$E_{2}$ : we exploit the discourse segmentation in Fig. 3 and consider the frequency of laughs in topic transition segments (wo) and topic continuation segments (wi). 


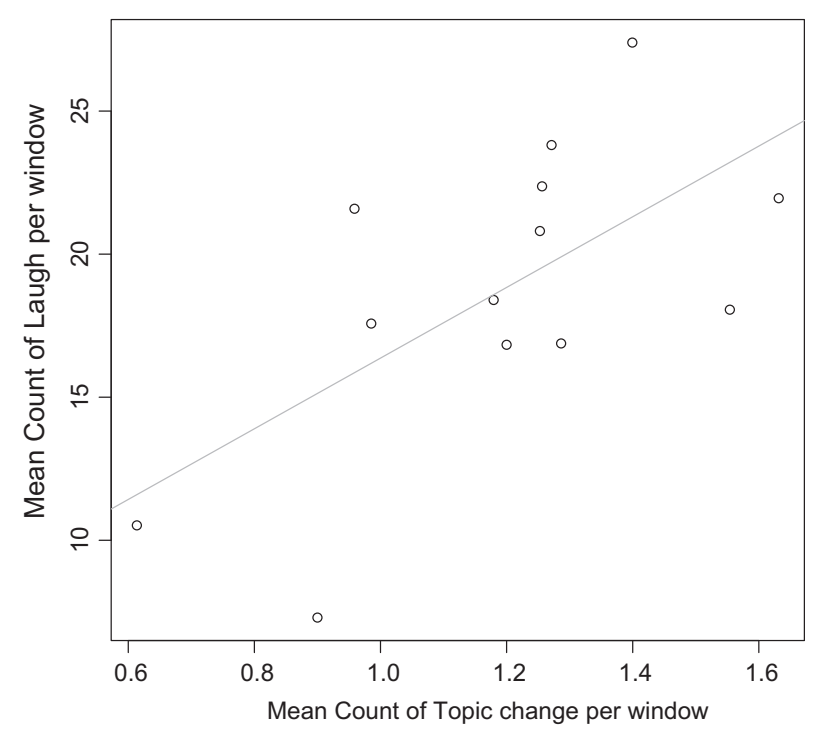

Fig. 6. Correlation between frequency of laughter and frequency of topic changes.

Description of $\boldsymbol{E}_{1}$. We consider the distance between last laugh and topic change and topic change and first laugh. We notice that those distances are not normally distributed. This results is confirmed on both our corpora. As shown in [9], analysis of TableTalk shows that LLs tend to occur at a shorter temporal distance from the T-event, than FLs: $\mu(\mathrm{LT})<\mu(\mathrm{TL})^{7}$ The temporal distance between the last laugh of a topic and topic boundary, is significantly shorter than the temporal distance between the topic boundary and the first laugh, and Fig. 7 shows this difference in distributions. ${ }^{8}$ From the parallel analysis of these two corpora, an interesting finding emerges: laughter is more likely as the temporal distance from the topic boundary increases. Although the two corpora present a similar behavior (see Fig. 8), it is worth noticing the difference in the distance between laughs and topic boundaries. In TableTalk the first laugh after a topic change happens (median value) around $27 \mathrm{~s}$ after the beginning of a topic, while in AMI after $30 \mathrm{~s}$. The last laugh tends to happen around $9 \mathrm{~s}$ before the end of a topic in TableTalk, and around $26 \mathrm{~s}$ before the end of a topic in AMI. Although aware of the gross nature of the median, those results may be due to the fact that TableTalk is characterized by shorter topics and a more dynamic and unstructured exchange than AMI.

Description of $\boldsymbol{E}_{2}$. We consider the discourse segmentation described in Fig. 3, that distinguish between topic transition segment wo and topic continuation segments wi. We notice a significant difference in the distribution of laughter in wi and wo, where the average frequency of laughs in wo is significantly greater than the average frequency of laughs in wi ( $p$-value $<0.005){ }^{9}$

Ramification. From $\mathrm{E}_{1}$ it emerges that laughter is more likely as the temporal distance from the topic boundary increases. This finding is not sufficient to support the fact that laughter can be considered, in isolation, a valid topic termination cue, but suggests that laughs are more likely to occur at the topic terminations, rather than immediately after a topic change (at the topic onset). The particular distribution of laughter emerged from the two corpora underlies a discourse function of laughter which could be useful information in automatic topic boundary detection (cf. [5]).

\footnotetext{
${ }^{7}$ One tail wilcox.test, $\mathrm{mu}=0$, alternative less: $p$-value $\ll 0.005$. $\mathrm{R}$ function [28].

8 In Fig. 7, we report the logarithm of the distribution to emphasize differences visually. A one tailed Student's T-Test on the logarithm of the distribution is in line with the Wilcox test on the raw data.

${ }^{9}$ One tail wilcox.test, $\mathrm{mu}=0$, alternative less. $\mathrm{R}$ function [28].
}
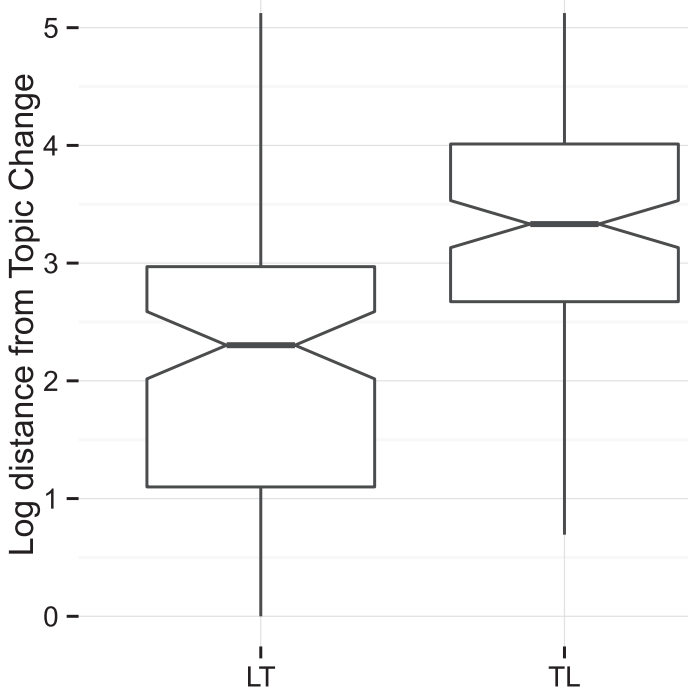

Fig. 7. $\mu(\mathrm{LT})$ vs. $\mu(\mathrm{TL})$ comparison in TableTalk.

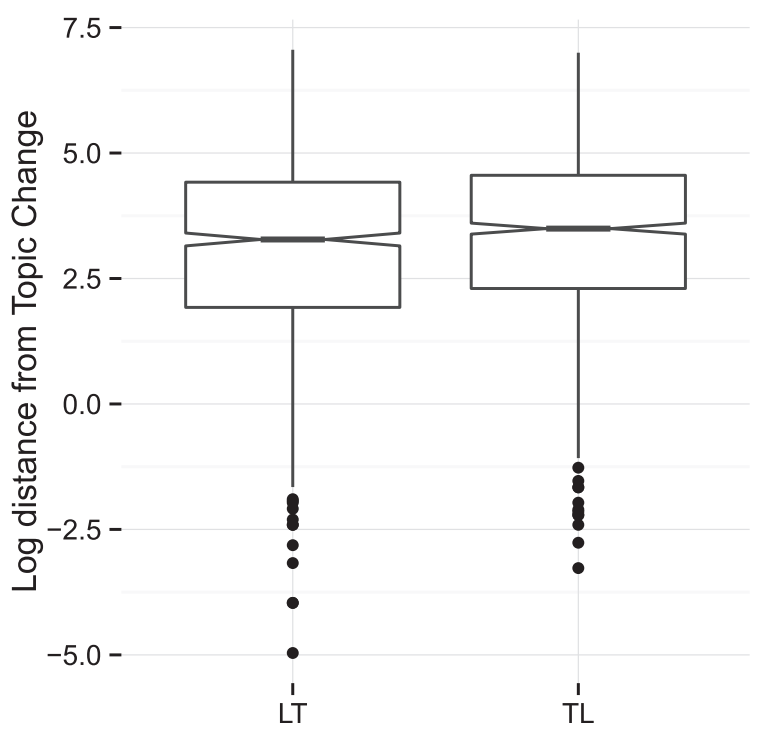

Fig. 8. $\mu(\mathrm{LT})$ vs. $\mu(\mathrm{TL})$ comparison in AMI.

From $E_{2}$ it emerges that topic continuation segments present fewer laughs than topic transition segments. This is in line with the positive correlation between frequency of laughs and amount of topic changes, described in Section 4.

It is reasonable to think that while a topic is discussed (topic continuation) a lot of topic-related information is transferred, and little space is left for social exchange. On the other hand, during the transition to a new topic, the conversation becomes more dynamic, alternating the information on the new topic with small talk. In the latter situation, laughter appears to be more frequent.

\subsubsection{Shared laughter and topic termination}

Having considered the laugh distribution at a coarse grain level, in this section we refine our analysis exploring the temporal distribution of shared and solo laughs with respect to topic changes. In the following we examine:

(a) Distribution of shared/solo laughter at topic terminations.

(b) Distribution of shared solo laughter in topic continuation vs. topic transition moments. 
In order to investigate (a) and (b), we consider previous studies [29], that explore similar distributions in a telephone conversation corpus of English native speakers. Holt ([3]) proposes a correlation between shared laughs and topic termination sequences. According to this analysis [3] shared laughs may be part of a topic termination sequence and may introduce to the end of the topic. The mutual acceptance of a laugh relates to the common agreement of a completed topic. Hence, we analyze whether, in our corpora, we find evidence of shared laughter being closer to the end of the topic than solo laughter. We refine our previous analysis of $\mu(\mathrm{LT})$ vs. $\mu(\mathrm{TL})$, distinguishing shared (SH) vs solo (SO) laugh. Since we are interested only in the topic termination section, we focus on the topic boundary left neighborhood $(\mu(\mathrm{LT}))$ and we explore the distance between shared laughs ( $\mathrm{SH})$ and topic change $(\mu(\mathrm{ShLT}))$ and solo laugh (SO) and topic change $(\mu($ SoLT $))$. As shown in Fig. 9, in TableTalk, some evidence is found of shared laughs being closer than solo laughs to topic termination boundaries, but this tendency does not reach significance. In particular, we notice how the median distance of a $\mathrm{SH}$ from topic termination is of $7 \mathrm{~s}$, while a median distance of SO from topic termination is of $12 \mathrm{~s}$. This result is different from that reported in initial work [9], because of the annotation differences in that work and the present analysis, as described above (Section 2.4). This clarifies that if moments of laughter separated by an interval are ever to be regarded as shared, then more needs to be learned about the constraints on those intervals, including the maximum interval length. The setting originally used [9], one second distance, was intuitively and empirically well justified, in our view, and using that setting, the difference described here becomes significant. However, in what remains we retain the constraint that only temporally overlapping laughs count as moments of shared laughter.

A similar behavior is found in AMI. We compare $\mu($ ShLT) and $\mu$ (SoLT), finding that $\mathrm{SH}$ do not tend to occur more in proximity of the end of the topic than SO (no significant difference in the distributions). This is shown in Fig. $10 .{ }^{10}$ In the AMI corpus, the median distance of $\mathrm{SH}$ and SO from topic termination is $28 \mathrm{~s}$. and $30 \mathrm{~s}$. respectively. Therefore, differently from previous studies, in these corpora topic termination sequences do not appear to be characterized by shared laughter more than by solo laughter.

\subsubsection{Distribution of solo laughter in topic continuation and topic transition segments}

In order to investigate (b), we analyse the SO and SH distribution in topic continuation segments, wi and topic transition segments, wo (refer to Fig. 3). It had been observed that solo laughter may be tied to topic continuation moments [29]. Recalling the observation that laughter can invite reciprocal laughter [30], Holt [29] interprets solo laughter as rejected invitations that happen when the recipient wants to add information and continue the topic. If solo laughs are related to topic continuation, we would expect a greater number of solo laughs in topic continuation segments (wi), rather than in topic transition segments (wo). We look for evidence of this observation in both corpora. In contrast to what we expect, it emerges that both TableTalk and AMI present a significant higher presence of SO laughs in topic transition moments, wo, rather than in topic continuations, wi, as evident in Figs. 11 and 12. As our null hypothesis we assume SOwi $\geqslant$ SOwo. From the analysis of the distributions, we can reject the null hypothesis for both corpora, in favor of the alternative hypothesis SOwi < SOwo. ${ }^{11}$ Solo

\footnotetext{
10 Fig. 10, we report the logarithm of the distribution to emphasize differences visually. One tailed Student's T-Test on the logarithm of the distribution is in line with the Wilcox test on the raw data.

$11 \mathrm{H} 1$ : SOwi $<$ SOwo, one tail wilcoxon test $\mathrm{mu}=0$, alternative less, $p$-value $<0.05$ in TableTalk and $p$-value $<0.0005$ in AMI. R function [28].
}

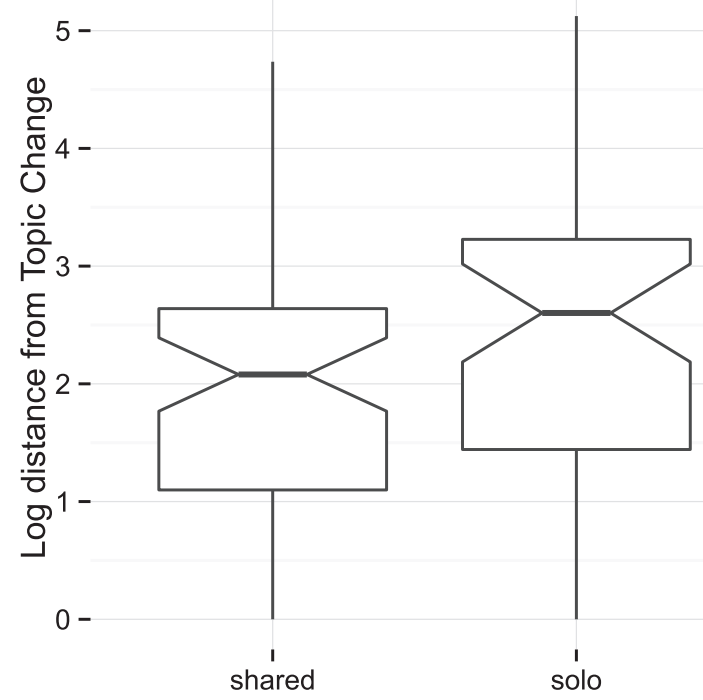

Fig. 9. $\mu$ (ShLT) and $\mu$ (SoLT) in TableTalk.

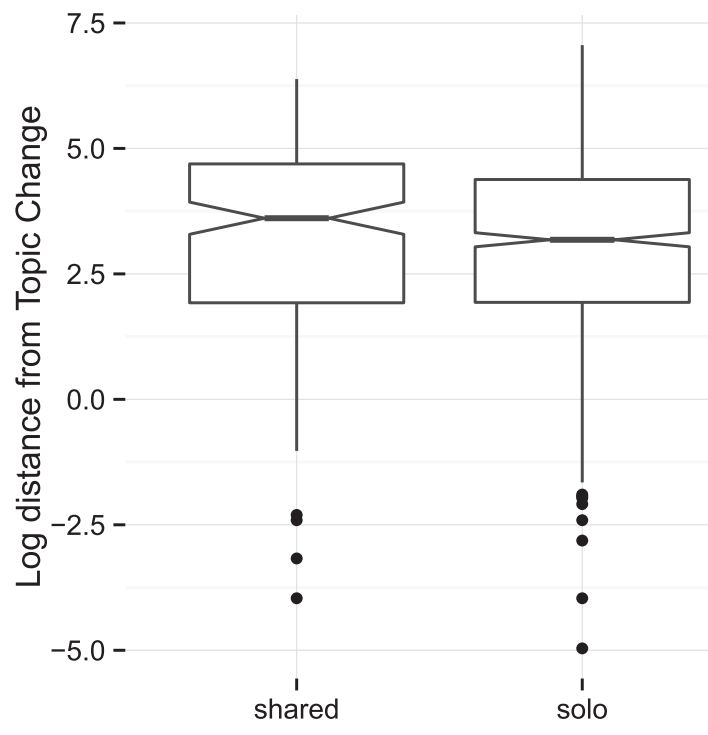

Fig. 10. $\mu($ ShLT $)$ and $\mu($ SoLT $)$ in AMI.

laughter appears to be more frequent in topic transition than in topic continuations.

\subsection{Laughter \& topic: content distribution}

In this section we address the relation between laughter, topic changes and information flow: How does the information flow vary in topic termination and topic beginning segments? In order to answer this question, we take the last laugh and the first laugh as landmarks for determining topic termination segments and topic beginning segments (LT and TL of Fig. 1), and we explore the distribution of information in those segments. We base our analysis of the information flow on the lexical richness of the segment, and we rely on the type token ratio (TTR) measure normalized over the length of the segment, as in (3). ${ }^{12}$ We calculate $\Lambda()$ over $\mathrm{LT}$ and $\mathrm{TL}$, having $\Lambda(\mathrm{LT})$ representing the lexical richness at

\footnotetext{
12 For any segment, the total number of unique words divided by total number of words - the value for this footnote is $\frac{21}{29}$ for all words, $\frac{12}{16}$ on only content.
} 


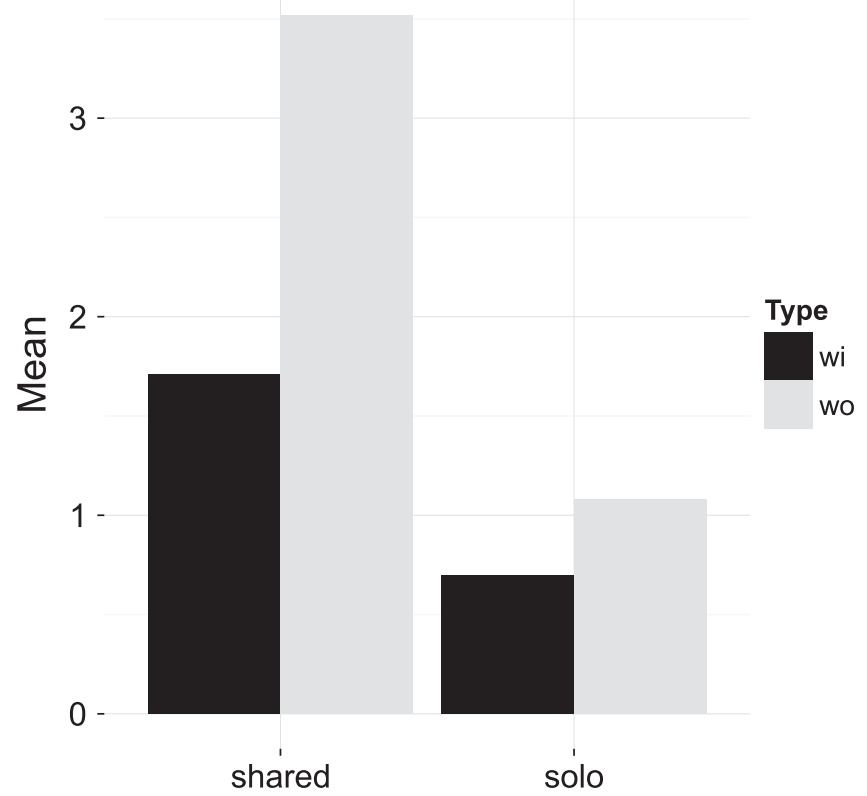

Fig. 11. Distribution of SO laughs in wi and wo segments in TableTalk corpus.

topic termination and $\Lambda(\mathrm{TL})$ the richness variety at topic beginning. Interestingly, we observe the same, unexpected trend both in TableTalk and in the AMI corpus. Topic termination exchange segments, $\Lambda$ (LT) show higher lexical richness than topic beginning segments $\Lambda(\mathrm{TL})$, although the latter should introduce, by definition, a new topic in conversation.

The distribution of $\Lambda(\mathrm{LT})$ and $\Lambda(\mathrm{TL})$ for TableTalk is shown in Fig. 13, while for AMI in Fig. 14. In both datasets, the null hypothesis, $\Lambda(\mathrm{LT}) \leqslant \Lambda(\mathrm{TL})$, is rejected. ${ }^{13}$

However, as we noticed in Section 5, LT and TL segments have a significant difference in length. Hence, there is argument for thinking that, in addition to the Class (LT vs. TL), also the factor Length of the segments could influence the differences in lexical richness. In fact, it is a fact of language that is more likely the presence of repetitions in longer segments than in shorter segments ${ }^{14}$ independently of whether the segments are individuated by laughter and topic changes.

Therefore, in order to verify the effect of these two factors in isolation, we look at the proportions between number of Types and the number of Repetitions (Tokens-Types) per segment Fig. 15. We created a generalized linear model in order to understand the correlation between the proportions (Types and Repetitions) and the variables Class and Length, considered as two independent variables. ${ }^{15}$ It emerges that both Length and Class have a significant independent effects on the proportion (Types, Repetitions).

However, we know that Length and Class are strongly correlated (see 5.1) in the sense that LT segments tend to be shorter than TL segments). Given this correlation, we cannot exclude the possibility that the effect of Class is entirely contained in the effect of the Length. To explore this, we created again two generalized linear models: one modeling the interaction of Length on the proportion (Types, Repetitions), and one modeling the independent interaction of Class and Length on the proportion (Types,

\footnotetext{
13 One tail wilcox.test, $\mathrm{mu}=0$, alternative greater: $p$-value $\ll 0.005$. $\mathrm{R}$ function [28]

14 Therefore, longer segments are more likely to have a lower TTR than shorter segments.

${ }^{15}$ In R: glm ((Types, Repetitions) Class + Length).
}

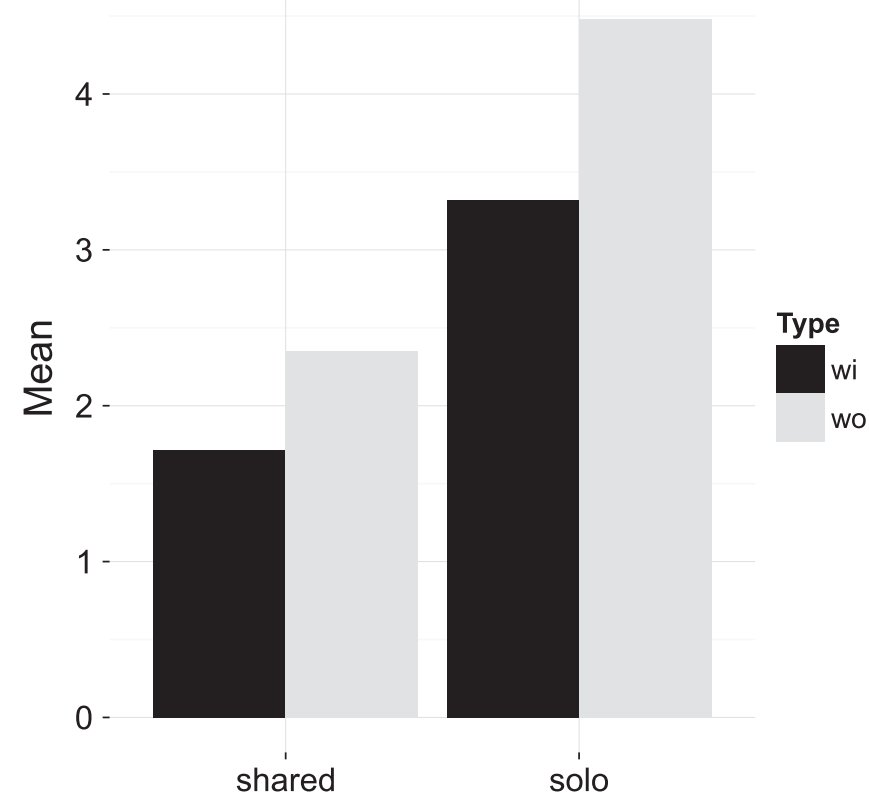

Fig. 12. Distribution of SO laughs in wi and wo segments in AMI corpus.

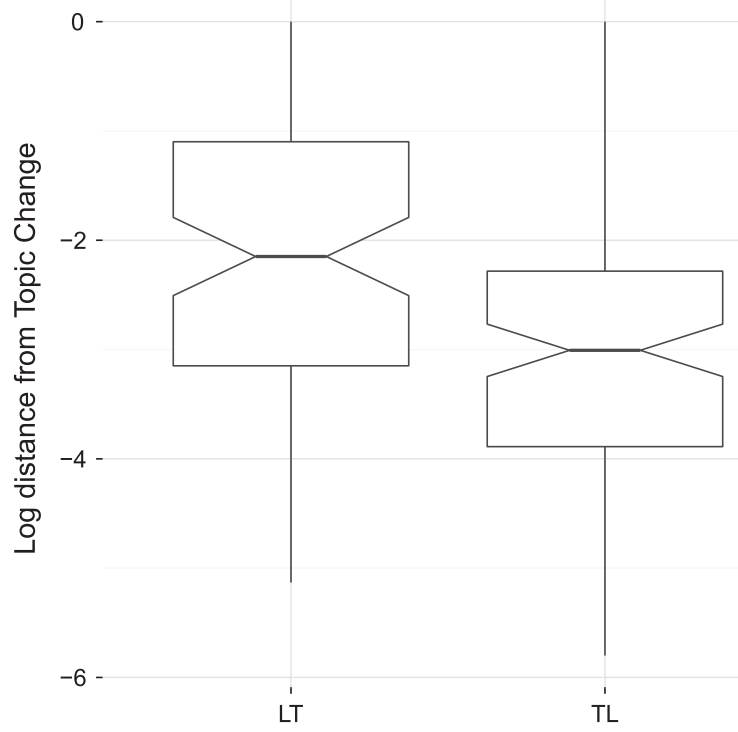

Fig. 13. $\Lambda(\mathrm{LT})$ vs. $\Lambda(\mathrm{TL})$ in TableTalk.

Repetitions). A difference among those models reflects the fact that Class has an independent influence on the proportion. A lack of significant difference in these models reflects the fact that the effect of the Class is entirely due to the difference in Length among the LT and TL.

Analysis of variance reveals that these two models are significantly different from the model with both terms as independent factors. Hence we conclude that the effect of Class is not just a coarse grain generalization of Length, but it has an independent effect on the lexical variety of LT and TL.

\section{Discussion}

Results show interesting similarities in the overall laughter distribution between the two corpora, despite the different nature 


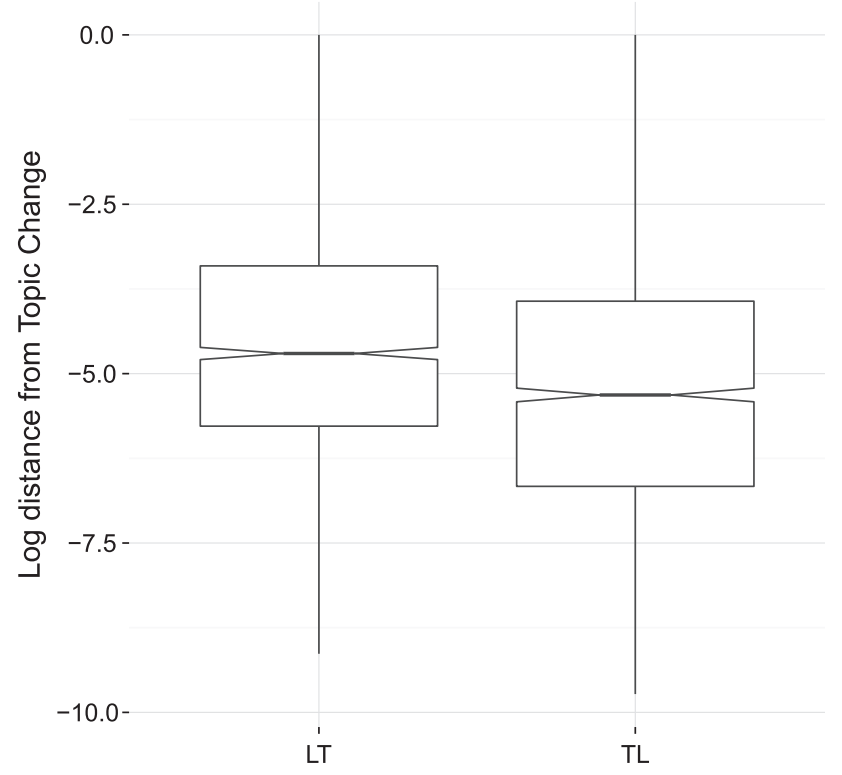

Fig. 14. $\Lambda(\mathrm{LT})$ vs. $\Lambda(\mathrm{TL})$ in AMI corpus.

of the corpora. While TableTalk is constituted of more unstructured conversations (mainly social interaction), in AMI moments of social interaction alternate with task oriented dialog moments [31]. Interestingly, in both corpora some patterns in the laughter distribution are found. First of all segments of topic transitions show higher presence of laughter than segments of topic continuation (see Exp. 2). In addition, looking at the topic termination moments vs. topic beginnings, it is evident that laughter tends not to occur immediately after a topic change (i.e., at the topic onset).

Although laughter in isolation is not a sufficient indication of topic change, this information (that laughter less likely to occur at the topic onset) can be use as a feature to enhance topic boundaries detection.

Regarding the distribution of shared laughter, in contrast with previous observations by Holt [29], we do not find any significant difference in their behavior around topic terminations. Both shared and solo laughter are equally likely to appear in topic termination moments. In the same work, [29], the author also observes how solo laughter may signal the continuation of a topic. Our results do not confirm this statement, as solo laughter is found to be more likely in topic transition moments rather than in topic continuations.

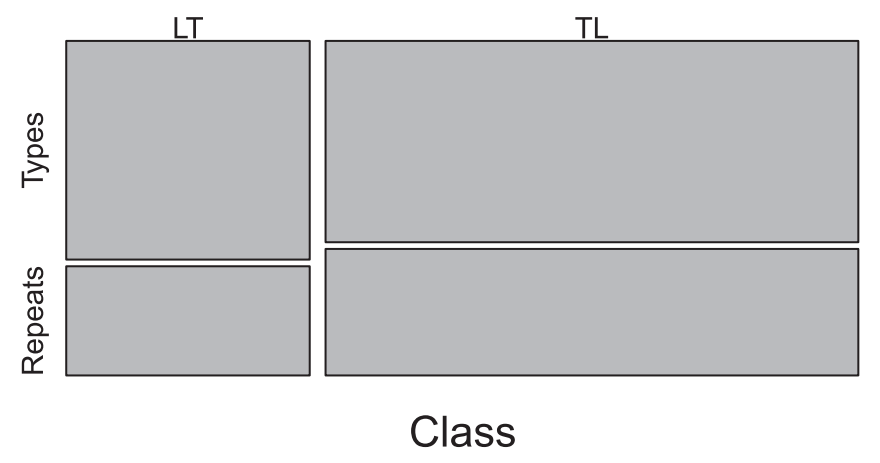

Fig. 15. Proportion of Types and Repetitions per Class, where Repetitions = TokensTypes.
Finally, in both corpora, we notice that, taking laughter as a discourse marker determining topic termination segments and topic onsets, the former tend to have higher lexical richness then the latter. Considering lexical richness as our measure of information flow, we notice that topic onsets and topic termination differ. We can then conclude that laughter and topic changes define segments of conversation which have a consistently different amount of information, hence laughter, in this case, serves a demarcation function. A possible interpretation of this phenomenon may stand in the grounding effect. In spoken interaction, participants have been observed to adapt their speech production to that of their interlocutor [32]. This alignment is usually a long term phenomenon, evolving during the conversation. However, from a qualitative analysis, an increase of lexical alignment (grounding) at the topic beginnings can be noticed; participants tend to establish the lexical common ground on what they are going to discuss. An example of this is given in the following extract, taken from a topic beginning in TableTalk:

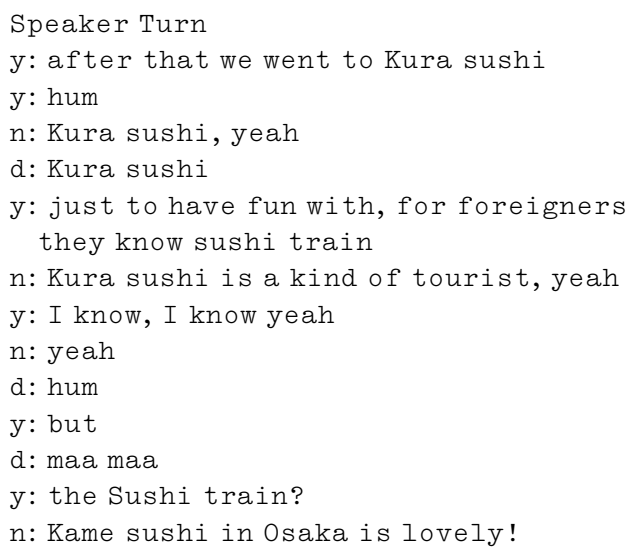

\section{Conclusions}

We examined the discourse function of laughter, investigating whether laughs can signal structural development of conversation, such as topic changes. We explored laughter timing with respect to topic changes and the dynamics of the information flow around topic changes and laughter. Results lead to the conclusion that laughter has quantifiable discourse functions alongside social signaling capacity.

Although laughter, in isolation, cannot be considered a reliable indicator of topic changes, it can contribute (with other features) to marking possible development in conversations. In the appendix, we report excerpts of laughter in relation to topic changes.

Finally we notice differences in the information flow between topic termination and topic beginnings. This result strengthens the hypothesis of the discourse function of laughter. Future work will be dedicated to investigate this latter finding, to explore the functions of other kind of social signals, and to investigate possible robust solutions for shared laughter annotation capable to handle annotation flaws.

\section{Acknowledgments}

This work is supported by the Innovation Bursary of Trinity College Dublin, the Speech Communication Lab at TCD, and by the SFI FastNet project 09/IN.1/1263. 


\section{Appendix A}

Below is an excerpt, from the AMI corpus, where share laughter anticipates a topic change from the AMI corpus:

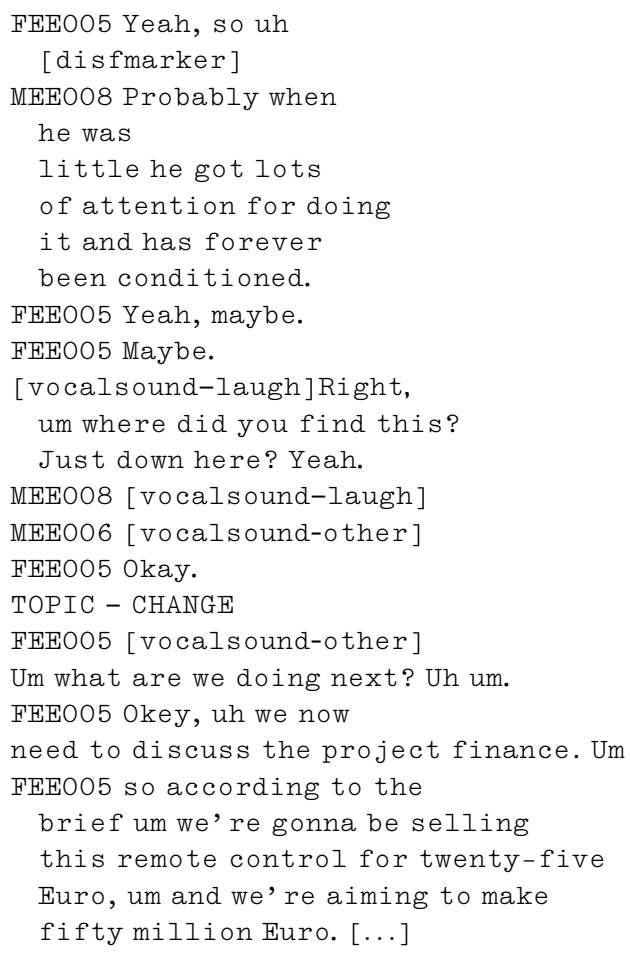

Below an excerpt, from the AMI corpus, in which laughter does not anticipate a topic change:

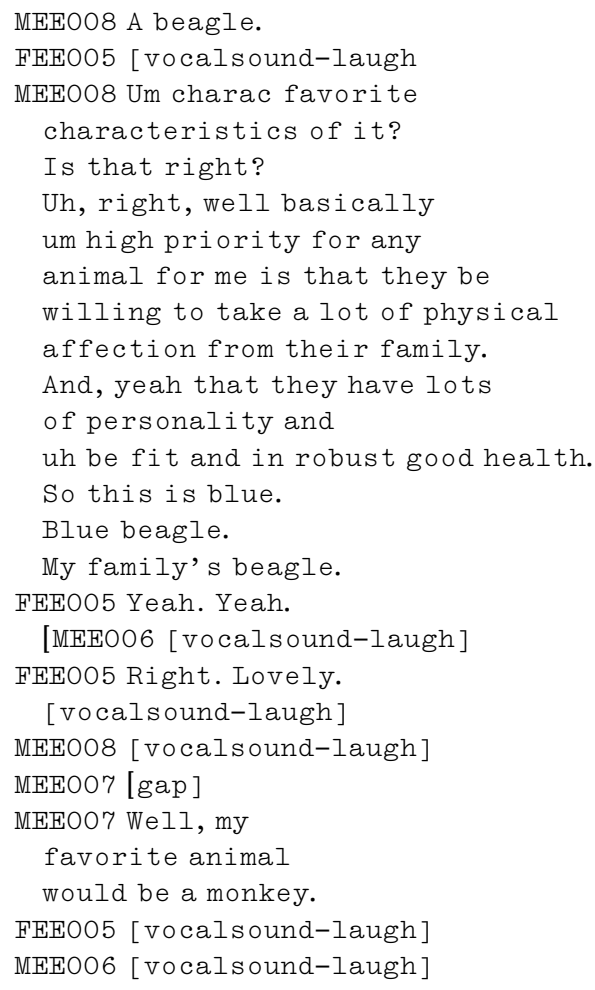

\section{Below an excerpt showing repetition at topic beginnings:}

FEE005 Is that what everybody got? Okay.

Um. So we' re gonna

have like individual

work and then

a meeting about it.

And repeat that process

three times.

Um and at this point

we get try out the

whiteboard over there.

Um. [vocalsound] So uh you get

to draw your

favorite animal and sum up your

favorite

characteristics of it.

So who would like to go first?

MEEOO' Yeah.

MEE008 Yeah.

TOPIC - CHANGE

MEE008 I will go. That's fine.

FEEO05 Very good. [vocalsound]

MEE008 Alright. So [disfmarker]

MEEO08 [vocalsound] [vocalsound]

MEE008 This one here

FEEO05 Mm-hmm.

MEEO08 Okay. Very nice.

Alright. My favorite animal

MEEOO8 is like [disfmarker]

MEEO08 [vocalsound]

MEEO08 [vocalsound]

\section{References}

[1] J.A. Bea, P.C. Marijuán, The informational patterns of laughter, Entropy 5 (2) (2003) 205-213.

[2] W. Ruch, J. Hofmann, T. Platt, Investigating facial features of four types of laughter in historic illustrations: why is conversation so easy?, Eur J. Humour Res. 1 (1) (2013) 99-118.

[3] E. Holt, On the nature of 'laughables': laughter as a response to overdone figurative phrases, Pragmatics 21 (3) (2011) 393-410. <http://eprints. hud.ac.uk/11553/>.

[4] M. Kurematsu, M. Ohashi, O. Kinosita, J. Hakura, H. Fujita, A study of how to implement a listener estimate emotion in speech, in: SoMeT, 2009, pp. 531540.

[5] S. Luz, The nonverbal structure of patient case discussions in multidisciplinary medical team meetings, ACM Trans. Inf. Syst. 30 (3) (2012) 17.

[6] S. Luz, J. Su, The relevance of timing, pauses and overlaps in dialogues: detecting topic changes in scenario based meetings, in: INTERSPEECH, 2010, pp. 1369-1372.

[7] S. Maskey, J. Hirschberg, Summarizing speech without text using hidden markov models, in: Proceedings of the Human Language Technology Conference of the NAACL, Companion Volume: Short Papers, NAACL-Short '06, Association for Computational Linguistics, Stroudsburg, PA, USA, 2006, pp. 89-92.

[8] N. Campbell, An audio-visual approach to measuring discourse synchrony in multimodal conversation data, in: Proceedings of Interspeech 2009, 2009, pp. 2159-2162.

[9] F. Bonin, N. Campbell, C. Vogel, Laughter and topic changes: temporal distribution and information flow, in: Cognitive Infocommunications (CogInfoCom), 2012 IEEE 3rd International Conference, 2012, pp. 53-58.

[10] I. Mccowan, G. Lathoud, M. Lincoln, A. Lisowska, W. Post, D. Reidsma, P. Wellner, The ami meeting corpus, in: L.P.J.J. Noldus, F. Grieco, L.W.S. Loijens, P.H. Zimmerman (Eds.), Proceedings Measuring Behavior 2005, 5th International Conference on Methods and Techniques in Behavioral Research, Noldus Information Technology, Wageningen, 2005.

[11] E. Schegloff, Sequence organization in interaction, A Primer in Conversation Analysis, vol. 1, Cambridge University Press, 2007.

[12] E. Keenan, Towards a universal definition of 'subject', in: C. Li (Ed.), Subject and Topic, Symposium on Subject and Topic, University of California, Santa Barbara, Academic Press, London, 1975, pp. 304-333. 
[13] K. Lambrecht, Information Structure and Sentence Form: Topic, Focus, and the Mental Representations of Discourse Referents, in: Cambridge Studies in Linguistics, Cambridge University Press, 1996.

[14] T.A. Van Dijk, Sentence topic versus discourse topic, Mouton (1981) 177194.

[15] T. Van Dijk, Discourse, power and access, in: C.R. Caldas-Coulthard, M. Coulthard (Eds.), Texts and Practices, Readings in Critical Discourse Analysis, Routledge, 1996, pp. 84-104.

[16] R.J. Passonneau, D.J. Litman, Discourse segmentation by human and automated means, Comput. Linguist. 23 (1) (1997) 103-139.

[17] M.A. Hearst, Texttiling: segmenting text into multi-paragraph subtopic passages, Comput. Linguist. (1997) 33-64.

[18] J.C. Reynar, An automatic method of finding topic boundaries, in: ACL, 1994, pp. 331-333.

[19] L. Sidner, B. Grosz, Attention, Intentions, and the Structure of Discourse, University of Illinois at Urbana-Champaign, 1986

[20] K.P. Truong, J. Trouvain, Laughter annotations in conversational speech corpora-possibilities and limitations for phonetic analysis, in: Proceedings of the 4th International Worskhop on Corpora for Research on Emotion Sentiment and Social Signals, 2012, pp. 20-24.

[21] K. Jokinen, Gaze and gesture activity in communication, in: C. Stephanidis (Ed.), Universal Access in Human-Computer Interaction. Intelligent and Ubiquitous Interaction Environments, of Lecture Notes in Computer Science, vol. 5615, Springer, Berlin/Heidelberg, 2009, pp. 537-546.

[22] J. Allwood, L. Cerrato, K. Jokinen, C. Navarretta, P. Paggio, The mumin coding scheme for the annotation of feedback, turn management and sequencing phenomena, Language Resour. Evaluat. 41 (3-4) (2007) 273287.
[23] F. Bonin, R. Böck, N. Campbell, How do we react to context? annotation of individual and group engagement in a video corpus, in: SocialCom/PASSAT, 2012, pp. 899-903.

[24] E. Gilmartin, F. Bonin, C. Vogel, N. Campbell, Laughter and topic transition in multiparty conversation, in: Proceedings of the SIGDIAL 2013 Conference, August 2013, Metz, France, pp. 304-308.

[25] E. Gilmartin, F. Bonin, N. Campbell, C. Vogel, Exploring the role of laughter in multiparty conversation, in: Proceedings of the SemDial 2013 (DialDam), Amsterdam, Netherlands, December 2013, pp. 191-193.

[26] C. Vogel, L. Behan, Measuring synchrony in dialog transcripts, in: A. Esposito, A.M. Esposito, A. Vinciarelli, R. Hoffmann, V.C. Müller (Eds.), 7403 Cognitive Behavioural Systems, Springer, LNCS, 2012, pp. 73-88.

[27] K. Toutanova, C.D. Manning, Enriching the knowledge sources used in a maximum entropy part-of-speech tagger, in: Proceedings of the Joint SIGDAT Conference on Empirical Methods in Natural Language Processing and Very Large Corpora (EMNLP/VLC-2000), 2000, pp. 63-70.

[28] M. Hollander, D.A. Wolfe, Non Parametric Statistical Methods, Wiley, 1999.

[29] E. Holt, The last laugh: shared laughter and topic termination, J. Pragmat. 42 (6) (2010) 1513-1525.

[30] G. Jefferson, A technique for inviting laughter and its subsequent acceptance/ declination, in: G. Psathas (Ed.), Everyday Language: Studies in Ethnomethodology, Irvington Publishers, New York, NY, 1979, pp. 79-96.

[31] T. Bickmore, J. Cassell, How about this weather? Social dialogue with embodied conversational agents, in: K. Dautenhahn (Ed.), Socially Intelligent Agents: The Human in the Loop (Papers from the 2000 AAAI Fall Symposium), 2000, pp. 4-8

[32] S. Garrod, M.J. Pickering, Why is conversation so easy?, Trends Cognitive Sci 8 (1) (2004) 8-11. 